\title{
Supplementary Materials: Joint Bayesian analysis of multiple response-types using the hierarchical generalized transformation model
}

Jonathan R. Bradley*

\section{Appendix A: Terminology}

\begin{tabular}{|c|c|c|}
\hline Terminology & Notation & Additional Comments \\
\hline Multiple response-type data & $\begin{array}{c}Z_{i j} \text { for } i=1, \ldots, I \text { and } j=1,2,3 \\
\mathbf{z}_{t r n}=\left(Z_{i j}: i=1, \ldots, I_{j}, j=1,2,3\right)^{\prime} \\
\mathbf{z}_{v a l}=\left(Z_{i j}: i=I_{j}+1, \ldots, I_{j}^{v a l}, j=1,2,3\right)^{\prime}\end{array}$ & $\begin{array}{l}\text { The index } i=1, \ldots, I_{j} \text { indicate that the } \\
\text { data is used for training, the index } i=I_{j}+ \\
1, \ldots, I_{j}^{\text {val }} \text { indicate that the data is used for } \\
\text { validation, and the index } i=I_{j}^{\text {val }}+1, \ldots, I \\
\text { indicate that the data is used for testing. }\end{array}$ \\
\hline Transformation & $h_{i j}$ for $i=1, \ldots, I_{j}$ and $j=1,2,3$ & $\begin{array}{l}\text { In Section } 2 \text { of the main text we also use } \\
\text { a functional notation } h_{i j}=h_{j}\left(Z_{i j}\right) \text { so that } \\
\text { one can more clearly see } h_{i j} \text { as a transfor- } \\
\text { mation. We drop this functional notation for } \\
\text { ease of exposition. }\end{array}$ \\
\hline Data model & $f\left(Z_{i j} \mid h_{i j}\right)$ for $i=1, \ldots, I$ and $j=1,2,3$ & $\begin{array}{l}\text { This pdf represents the conditional distri- } \\
\text { bution of the data } Z_{i j} \text { given the transfor- } \\
\text { mation } h_{i j} \text {. }\end{array}$ \\
\hline
\end{tabular}

Table 1: This paper contains several terms. The above contains a non-exhaustive list of such terms to help with organizing the terms. This table continues onto the next page.

* Department of Statistics, Florida State University, 117 N. Woodward Ave., Tallahassee, FL, 323064330 jrbradley@fsu.edu 
2 Supplementary Materials: hierarchical generalized transformation model

\begin{tabular}{|c|c|c|}
\hline Terminology & Notation & Additional Comments \\
\hline Transformation Data Model & $\begin{array}{c}f(\mathbf{h} \mid \mathbf{y}, \boldsymbol{\theta}) m(\mathbf{h} \mid \boldsymbol{\gamma}), \text { where } \\
m(\mathbf{h} \mid \boldsymbol{\gamma})=f(\mathbf{h} \mid \gamma) / \iint f(\mathbf{h} \mid \mathbf{y}, \boldsymbol{\theta}) f(\mathbf{y} \mid \boldsymbol{\theta}) f(\boldsymbol{\theta}) d \mathbf{y} d \boldsymbol{\theta}\end{array}$ & $\begin{array}{l}\text { The HGT defines two conditional distribu- } \\
\text { tions on } \mathbf{h} \text { : the transformation data model } \\
f(\mathbf{h} \mid \mathbf{y}, \boldsymbol{\theta}, \gamma)=f(\mathbf{h} \mid \mathbf{y}, \boldsymbol{\theta}) m(\mathbf{h} \mid \gamma) \text { and the } \\
\text { transformation prior } f(\mathbf{h} \mid \gamma) \text {. The presence } \\
\text { of } m(\mathbf{h} \mid \gamma) \text { forces these conditional densities } \\
\text { to have the same marginal distribution for } \\
\mathbf{h} \text {. This is a term in the "preferred model." }\end{array}$ \\
\hline The Latent Process & $\begin{array}{c}Y_{i j} \text { for } i=1, \ldots, I, j=1,2,3 \\
\mathbf{y}=\left(Y_{i j}=i=1, \ldots, I, j=1,2,3\right)^{\prime}\end{array}$ & $\begin{array}{l}\text { In general, we are primarily interested in } \\
\text { performing inference on } \mathbf{y} \text {. In Section } 3.4 \text { of } \\
\text { the main text, } Y_{i j}=\mathbf{x}_{i j}^{\prime} \boldsymbol{\beta}+\mathbf{S}_{i j}^{\prime} \boldsymbol{\eta}+\xi_{i j} .\end{array}$ \\
\hline The Process Model & $f(\mathbf{y} \mid \boldsymbol{\theta})$ & This is a term in the "preferred model." \\
\hline Prior distribution & $f(\boldsymbol{\theta})$ and $f(\boldsymbol{\kappa})$ & $\begin{array}{l}\text { In general, the density of any parameter as- } \\
\text { sociated with } Y_{i j} \text { or } Z_{i j} \text { is called a prior. } \\
\text { This includes } \boldsymbol{\theta} \text { and } \boldsymbol{\kappa} \text {. In Section } 3.4 \text { of the } \\
\text { main text } \boldsymbol{\theta}=\left(\boldsymbol{\beta}^{\prime}, \sigma^{2}, \sigma_{\xi}^{2}, \sigma_{\eta}^{2}\right)^{\prime} \text { and in Sec- } \\
\text { tion } 4.2 \boldsymbol{\kappa}=\left(\kappa_{10}, \kappa_{20}, \kappa_{30}, \kappa_{11}, \kappa_{21}, \kappa_{31}\right)^{\prime} \text {. } \\
\text { The density } f(\boldsymbol{\theta}) \text { is a term in the "preferred } \\
\text { model." }\end{array}$ \\
\hline Transformation prior & $f(\mathbf{h} \mid \gamma)$ & $\begin{array}{l}\text { The data model and the transformation pri- } \\
\text { ors will be used to produce updates of } h_{i j} \\
\text { (i.e., Step } 2 \text { in Algorithm } 1 \text { in the main text } \\
\text { and Equation (3.10)). }\end{array}$ \\
\hline
\end{tabular}

Table 1: (Cont'd) This paper contains several terms. The above contains a nonexhaustive list of such terms to help with organizing the terms. This Table continues onto the next page. 


\begin{tabular}{|c|c|c|}
\hline Terminology & Notation & Additional Comments \\
\hline Transformation hyperprior & $f(\gamma)$ & 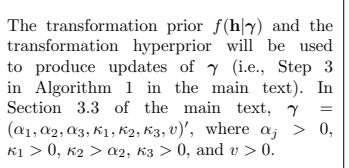 \\
\hline "is distributed as" & $\begin{array}{l}\text { Example of a named distribution: } Z_{Z} \mid h_{i} \text { ind } \\
\text { ind Normal }\left(h_{11}, v\right) \\
\text { Example of an un-named distributution: } \mathbf{y} \mid \boldsymbol{\theta} \sim f(\mathbf{\theta} \mid \boldsymbol{\theta})\end{array}$ & 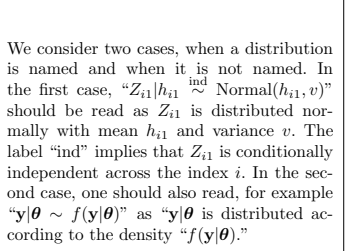 \\
\hline The preferred model & $\begin{aligned} \mathbf{h} \mid \mathbf{y}, \boldsymbol{\theta}, \boldsymbol{\theta}, \boldsymbol{\gamma} \sim f(\mathbf{h} \mid \mathbf{y}, \boldsymbol{\theta}) m(\mathbf{h} \mid \gamma) \\
\mathbf{y} \mid \boldsymbol{\theta} \sim f(\mathbf{y} \mid \boldsymbol{\theta}) \\
\boldsymbol{\theta} \sim f(\boldsymbol{\theta})\end{aligned}$ & 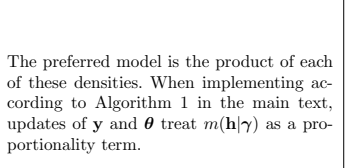 \\
\hline Posterior replicates & To denote replicates from a posterior distribution we use the superscript " (*") & 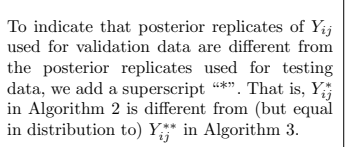 \\
\hline The generalized transformation model (HGT) & 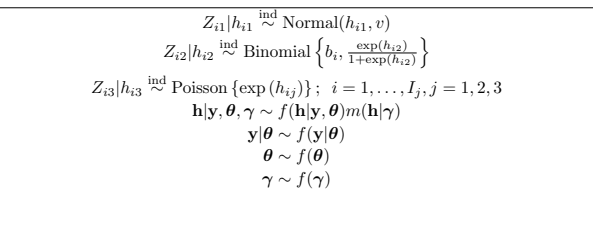 & $\begin{array}{l}\text { The HGT model is used for fitting training } \\
\text { data, while the GLIMM is used for fitting } \\
\text { validation and testing data. }\end{array}$ \\
\hline
\end{tabular}

Table 1: (Cont'd) This paper contains several terms. The above contains a nonexhaustive list of such terms to help with organizing the terms.

\section{Appendix B: Derivations}

Derivation of (3.6) in the Main Text: The distributions in (3.4) and (3.5) of the main text can be used to produce the following expression of the joint distribution of the 


\section{Supplementary Materials: hierarchical generalized transformation model}

data, process, and parameters

$$
f\left(\mathbf{z}_{t r n}, \mathbf{y}, \boldsymbol{\theta}\right)=\iint f\left(\mathbf{z}_{t r n} \mid \mathbf{h}\right) f(\mathbf{y}, \boldsymbol{\theta} \mid \mathbf{h}) f(\mathbf{h} \mid \gamma) f(\gamma) d \mathbf{h} d \boldsymbol{\gamma}=\int f(\mathbf{y}, \boldsymbol{\theta} \mid \mathbf{h}) f\left(\mathbf{z}_{t r n}, \mathbf{h}\right) d \mathbf{h},
$$

where $f\left(\mathbf{z}_{t r n}, \mathbf{h}\right)=\int f\left(\mathbf{z}_{t r n} \mid \mathbf{h}\right) f(\mathbf{h} \mid \gamma) f(\boldsymbol{\gamma}) d \boldsymbol{\gamma}$ and we have used the assumption of conditional independence between $\mathbf{z}_{t r n}$ and $(\mathbf{y}, \boldsymbol{\theta})$ given $\mathbf{h}$, and conditional independence of $\boldsymbol{\gamma}$ and $(\mathbf{y}, \boldsymbol{\theta})$ given $\mathbf{h}$. Then dividing by $f\left(\mathbf{z}_{t r n}\right)=\iint f\left(\mathbf{z}_{t r n} \mid \mathbf{h}\right) f(\mathbf{h} \mid \boldsymbol{\gamma}) f(\boldsymbol{\gamma}) d \mathbf{h} d \boldsymbol{\gamma}$ yields,

$$
f(\mathbf{y}, \boldsymbol{\theta} \mid \mathbf{z})=\int f(\mathbf{y}, \boldsymbol{\theta} \mid \mathbf{h}) f(\mathbf{h} \mid \mathbf{z}) d \mathbf{h},
$$

which is the desired result.

Derivation of (3.9) of the Main Text: Versions of this proof can be found in Diaconis and Ylvisaker (1979) and Bradley et al. (2020+). The two distributions in (3.7) of the main text associated with $j=2$ and $j=3$ are members of the natural exponential family (Lehmann and Casella, 1998), which are of the form,

$$
f\left(Z_{i j} \mid h_{i j}, \alpha_{j}, \kappa_{j}\right) \propto \exp \left\{Z_{i j} h_{i j}-c_{i j} \psi_{j}\left(h_{i j}\right)\right\} ; i=1, \ldots, I_{j}, j=2,3,
$$

where $c_{i 2}=b_{i}$ and $c_{i 3}=1$. Upon multiplying by (3.8) in the main text we have:

$f\left(h_{i j} \mid Z_{i j}, \alpha_{j}, \kappa_{j}\right) \propto \exp \left\{\left(Z_{i j}+\alpha_{j}\right) h_{i j}-\left(\kappa_{j}+c_{i j}\right) \psi_{j}\left(h_{i j}\right)\right\} \propto \mathrm{DY}\left(\alpha_{j}+Z_{i j}, \kappa_{j}+c_{i j} ; \psi_{j}\right)$,

which proves the result for $j=2$ and $j=3$. For $j=1$,

$$
\begin{aligned}
& f\left(h_{i 1} \mid Z_{i 1}, \alpha_{1}, \kappa_{1}\right) \propto \exp \left\{\left(\frac{Z_{i 1}}{v}+\alpha_{1}\right) h_{i 1}-\left(\kappa_{1}+\frac{1}{2 v}\right) h_{i j}^{2}\right\} \\
& =\exp \left\{2\left(2 \kappa_{1}+\frac{1}{v}\right)\left(2 \kappa_{1}+\frac{1}{v}\right)^{-1}\left(\frac{Z_{i 1}}{v}+\alpha_{1}\right) \frac{h_{i 1}}{2}-\left(2 \kappa_{1}+\frac{1}{v}\right) \frac{h_{i j}^{2}}{2}\right\} \\
& \propto \exp \left\{2\left(2 \kappa_{1}+\frac{1}{v}\right)\left(2 \kappa_{1}+\frac{1}{v}\right)^{-1}\left(\frac{Z_{i 1}}{v}+\alpha_{1}\right) \frac{h_{i 1}}{2}-\left(2 \kappa_{1}+\frac{1}{v}\right) \frac{h_{i j}^{2}}{2}\right. \\
& \left.-\frac{1}{2}\left(2 \kappa_{1}+\frac{1}{v}\right)\left(2 \kappa_{1}+\frac{1}{v}\right)^{-2}\left(\frac{Z_{i 1}}{v}+\alpha_{1}\right)^{2}\right\} \\
& =\exp \left[\frac{\left.\left\{h_{i 1}-\left(2 \kappa_{1}+\frac{1}{v}\right)^{-1}\left(\frac{Z_{i 1}}{v}+\alpha_{1}\right)\right\}^{2}\right]}{2\left(2 \kappa_{1}+\frac{1}{v}\right)^{-1}}\right] \\
& \propto \text { Normal }\left\{\left(2 \kappa_{1}+\frac{1}{v}\right)^{-1}\left(\frac{Z_{i 1}}{v}+\alpha_{1}\right),\left(2 \kappa_{1}+\frac{1}{v}\right)^{-1}\right\},
\end{aligned}
$$

which completes the results. 
Derivation of (3.11) in the Main Text: In Equation (14) we see that

$E\left(h_{i 1} \mid Z_{i 1}, \gamma\right)=\left(2 \kappa_{1}+\frac{1}{v}\right)^{-1}\left(\frac{Z_{i 1}}{v}+\alpha_{1}\right)+E\left(w_{1} \mid Z_{i 1}, \gamma\right)=\left(2 \kappa_{1}+\frac{1}{v}\right)^{-1}\left(\frac{Z_{i 1}}{v}+\alpha_{1}\right)$,

which converges to $Z_{i 1}$ as $\alpha_{1}$ and $\kappa_{1}$ approach zero. The expectation of a beta distribution is well known (Casella and Berger, 2002), which from (3.10) gives us

$$
E\left\{g\left(h_{i 2}\right) \mid Z_{i 2}, \gamma\right\}=E\left(w_{2} \mid Z_{i 1}, \gamma\right)=\frac{\alpha_{2}+Z_{i 2}}{\kappa_{2}+b_{i}},
$$

which converges to $Z_{i 2} / b_{i}$ as $\alpha_{2}$ and $\kappa_{2}$ approach zero. Similarly, the expectation of a gamma distribution is well known (Casella and Berger, 2002), which from (3.10) gives us

$$
E\left\{g\left(h_{i 3}\right) \mid Z_{i 3}, \gamma\right\}=E\left(w_{3} \mid Z_{i 1}, \gamma\right)=\frac{\alpha_{3}+Z_{i 3}}{\kappa_{3}+1},
$$

which converges to $Z_{i 3}$ as $\alpha_{3}$ and $\kappa_{3}$ approach zero.

Proof that (4.7) in the Main Text is Proper: The joint distribution of the training data, transformed data, process, parameters, and transformation hyperprior is given by:

$$
\left\{\prod_{i=1}^{I_{1}} f\left(Z_{i 1} \mid h_{i 1}\right)\right\}\left\{\prod_{i=1}^{I_{2}} f\left(Z_{i 2} \mid h_{i 2}\right)\right\}\left\{\prod_{i=1}^{I_{3}} f\left(Z_{i 3} \mid h_{i 3}\right)\right\} f(\mathbf{h} \mid \mathbf{y}, \boldsymbol{\theta}) m(\mathbf{h} \mid \boldsymbol{\gamma}) f(\mathbf{y} \mid \boldsymbol{\theta}) f(\boldsymbol{\theta}) f(\boldsymbol{\gamma}) .
$$

Then integrate out $\mathbf{y}$ and $\boldsymbol{\theta}$ to obtain,

$$
\left\{\prod_{i=1}^{I_{1}} f\left(Z_{i 1} \mid h_{i 1}\right)\right\}\left\{\prod_{i=1}^{I_{2}} f\left(Z_{i 2} \mid h_{i 2}\right)\right\}\left\{\prod_{i=1}^{I_{3}} f\left(Z_{i 3} \mid h_{i 3}\right)\right\}\left\{\prod_{i, j} f_{D Y}\left(h_{i j} \mid \alpha_{j}, \kappa_{j}, a, b\right)\right\} f(\gamma),
$$

which follows from,

$$
\begin{aligned}
& \iint f(\mathbf{h} \mid \mathbf{y}, \boldsymbol{\theta}) m(\mathbf{h} \mid \gamma) f(\mathbf{y} \mid \boldsymbol{\theta}) f(\boldsymbol{\theta}) d \mathbf{y} d \boldsymbol{\theta} \\
& =\iint f(\mathbf{h} \mid \mathbf{y}, \boldsymbol{\theta}) f(\mathbf{y} \mid \boldsymbol{\theta}) f(\boldsymbol{\theta}) d \mathbf{y} d \boldsymbol{\theta} \frac{\prod_{i, j} f_{D Y}\left(h_{i j} \mid \alpha_{j}, \kappa_{j}, a, b\right)}{\iint(f(\mathbf{h} \mid \mathbf{y}, \boldsymbol{\theta}) f(\mathbf{y} \mid \boldsymbol{\theta}) f(\boldsymbol{\theta}) d \mathbf{y} d \boldsymbol{\theta}}=\prod_{i, j} f_{D Y}\left(h_{i j} \mid \alpha_{j}, \kappa_{j}, a, b\right) .
\end{aligned}
$$

Finally, we have the result, since the normal, binomial, Poisson, and DY distributions (Diaconis and Ylvisaker, 1979) are proper and the prior on $\gamma$ is proper.

\section{Appendix C: Additional Model Details}

\section{Appendix C.1: Full-Conditional Distributions for the Transformation Hyperparameters}

The full-conditional distributions for the transformation hyperparameters and $v$ are found by multiplying $f(\mathbf{h} \mid \boldsymbol{\gamma})$ and $f(\boldsymbol{\gamma})$ as follows:

$$
v \mid \cdot \sim I G\left(\frac{I_{1}}{2}+a_{1}, \frac{\sum_{i=1}^{I_{2}}\left(Z_{i 1}-h_{i 1}\right)^{2}}{2}+b_{1}\right)
$$




$$
\begin{aligned}
& f\left(\alpha_{2} \mid \cdot\right) \propto \alpha_{2}^{a_{2}-1} \exp \left(-b_{2} \alpha_{2}\right) \frac{1}{\Gamma\left(\alpha_{2}\right)^{I_{2}} \Gamma\left(\kappa_{2}-\alpha_{2}\right)^{I_{2}}} \exp \left(\alpha_{2} \sum_{i=1}^{I_{2}} h_{i 2}\right) \\
& f\left(\alpha_{3} \mid \cdot\right) \propto \alpha_{3}^{a_{3}-1} \exp \left(-b_{3} \alpha_{3}\right) \frac{\kappa_{3}^{I_{3} \alpha_{3}}}{\Gamma\left(\alpha_{3}\right)^{I_{3}}} \exp \left(\alpha_{3} \sum_{i=1}^{I_{3}} h_{i 3}\right) \\
& f\left(\kappa_{2} \mid \cdot\right) \propto\left(\kappa_{2}-\alpha_{2}\right)^{\zeta_{2}-1} \exp \left(-\eta_{2} \kappa_{2}\right) \frac{\Gamma\left(\kappa_{2}\right)^{I_{2}}}{\Gamma\left(\kappa_{2}-\alpha_{2}\right)^{I_{2}}} \exp \left(-\kappa_{2} \sum_{i=1}^{I_{2}} \log \left(1+\exp \left(h_{i 2}\right)\right)\right) \mathcal{I}\left(\kappa_{3} \geq \alpha_{3}\right) \\
& \left.f\left(\kappa_{3} \mid \cdot\right) \propto\left(\kappa_{3}-\alpha_{3}\right)^{\zeta_{3}-1} \exp \left(-\eta_{3} \kappa_{3}\right) \kappa_{3}^{I_{3} \alpha_{3}} \exp \left(-\kappa_{3} \sum_{i=1}^{I_{3}} \exp \left(h_{i 3}\right)\right) \mathcal{I}\left(\kappa_{3} \geq \alpha_{3}\right), \quad \text { C.1.1 }\right)
\end{aligned}
$$

where $\Gamma(t)=\int_{0}^{\infty} x^{t-1} \exp (-x) d x, \mathcal{I}(\cdot)$ is the indicator function, and $I G(a, b)$ is an inverse gamma distribution with shape $a>0$ and rate $b>0$. In our implementation we set the parameters $a_{1}=a_{2}=a_{3}=\zeta_{2}=\zeta_{3} 1$ and $b_{1}=b_{2}=b_{3}=\eta_{2}=\eta_{3}=1$. We have found that our results are robust to this specification. Step 3 of Algorithm 1 involves simulating from the full conditional distributions in (C.1.1).

\section{Appendix C.2: Full-Conditional Distributions for the Spatio-Temporal Mixed Effects Model}

The full conditional distributions for this spatio-temporal mixed effects model are wellknown (e.g.,see Cressie and Wikle, 2011, for a standard reference reference) and are as follows:

$$
\begin{array}{ccc}
\boldsymbol{\beta} \mid \cdot \sim \operatorname{Normal}\left(\boldsymbol{\mu}_{\beta}^{*}, \boldsymbol{\Sigma}_{\beta}^{*}\right) ; & \boldsymbol{\mu}_{\beta}^{*} \equiv \frac{1}{\sigma^{2}} \boldsymbol{\Sigma}_{\beta}^{*}(\mathbf{h}-\boldsymbol{\xi}-\mathbf{S} \boldsymbol{\eta}), & \boldsymbol{\Sigma}_{\beta}^{*} \equiv\left(\frac{1}{\sigma^{2}} \mathbf{X}^{\prime} \mathbf{X}+\frac{1}{\sigma_{\beta}^{2}} \mathbf{I}_{p}\right)^{-1} \\
\boldsymbol{\eta} \mid \cdot \sim \operatorname{Normal}\left(\boldsymbol{\mu}_{\eta}^{*}, \boldsymbol{\Sigma}_{\eta}^{*}\right) ; & \boldsymbol{\mu}_{\eta}^{*} \equiv \frac{1}{\sigma^{2}} \boldsymbol{\Sigma}_{\eta}^{*}(\mathbf{h}-\mathbf{X} \boldsymbol{\beta}-\boldsymbol{\xi}), & \boldsymbol{\Sigma}_{\eta}^{*} \equiv\left(\frac{1}{\sigma^{2}} \mathbf{I}_{r}+\frac{1}{\sigma_{\eta}^{2}} \mathbf{I}_{r}\right)^{-1} \\
\boldsymbol{\xi} \mid \cdot \sim \operatorname{Normal}\left(\boldsymbol{\mu}_{\xi}^{*}, \boldsymbol{\Sigma}_{\xi}^{*}\right) ; & \boldsymbol{\mu}_{\xi}^{*} \equiv \frac{1}{\sigma^{2}} \boldsymbol{\Sigma}_{\xi}^{*}(\mathbf{h}-\mathbf{X} \boldsymbol{\beta}-\mathbf{S} \boldsymbol{\eta}), & \boldsymbol{\Sigma}_{\xi}^{*} \equiv\left(\frac{1}{\sigma^{2}} \mathbf{I}_{n}+\frac{1}{\sigma_{\xi}^{2}} \mathbf{I}_{n}\right)^{-1}
\end{array}
$$

The full conditional distributions for variance parameters are well-known (e.g.,see Gelman et al., 2013, for a standard reference) and are as follows:

$$
\begin{aligned}
\sigma^{2} \mid \cdot & \sim I G\left(\frac{n}{2}+\alpha_{v}, \frac{\sum_{i=1}^{I_{2}} \sum_{j=1}^{3}\left(h_{i 1}-\mathbf{x}_{i j}^{\prime} \boldsymbol{\beta}-\mathbf{S}_{i j}^{\prime} \boldsymbol{\eta}-\xi_{i j}\right)^{2}}{2}+\beta_{v}\right) \\
\sigma_{\eta}^{2} \mid \cdot & \sim I G\left(\frac{r}{2}+\alpha_{\eta}, \frac{\boldsymbol{\eta}^{\prime} \boldsymbol{\eta}}{2}+\beta_{\eta}\right) \\
\sigma_{\xi}^{2} \mid \cdot & \sim I G\left(\frac{n}{2}+\alpha_{\xi}, \frac{\boldsymbol{\xi}^{\prime} \boldsymbol{\xi}}{2}+\beta_{\xi}\right) .
\end{aligned}
$$


Step 4 of Algorithm 1 for this model involves simulating from the full-conditional distributions in (C.2.1) and (C.2.2).

\section{Appendix C.3: Bayesian Additive Regression Trees}

Consider the following expression for the BART model (e.g., see Chipman et al., 2010, among others):

Data Model : $h_{i j} \mid \mathbf{M}_{k}, \mathbf{T}_{k}, \sigma^{2}, \boldsymbol{\lambda} \stackrel{\text { ind }}{\sim}$ Normal $\left\{\sum_{k=1}^{m} w\left(\mathbf{x}_{i j} ; \mathbf{M}_{k}, \mathbf{T}_{k}\right), \sigma^{2}\right\} m(\mathbf{h} \mid \boldsymbol{\lambda})$;

Prior 1: $\mu_{g h} \mid \mathbf{T}_{k} \sim$ Normal $\left(0, \frac{1}{4 \epsilon^{2} m}\right)$;

Prior 2: $\sigma^{2} \sim \operatorname{IG}\left(\alpha_{v}, \beta_{v}\right)$;

Prior 3: $f\left(\mathbf{T}_{k}\right) \propto \prod_{g=1}^{u_{k}} \alpha\left(1+d_{g}\right)^{-\beta} ; i=1, \ldots I_{j}, j=1,2,3$,

where $\mathbf{x}_{i j}$ is a $p$-dimensional vector of known covariates, $w(\cdot)$ is a decision tree (see definition in Chipman et al., 2010), set $\mathbf{M}_{k}=\left(\mu_{11} \ldots \mu_{b_{k} k}^{\prime}, b_{k}\right.$ is the $k$-th terminal node, and $d_{k}$ is the depth of internal node $k$. The hyperparameters $\epsilon \in[1,3], \alpha_{\nu}>$ $0, \beta_{v}>0, \alpha>0$, and $\beta>0$ are chosen based on the default specifications of the $\mathrm{R}$ package BayesTree (Chipman and McCulloch, 2016). Implementation is achieved through a Metropolis-within-Gibbs sampler and a backfitting algorithm as described in Chipman et al. (2010). This Markov chain Monte Carlo (MCMC) algorithm is computed using the $\mathrm{R}$ package BayesTree. That is, Step 4 of Algorithm 1 for this model involves simulating from posterior distribution of $\left\{\mathbf{M}_{k}\right\},\left\{\mathbf{T}_{k}\right\}$, and $\sigma^{2}$ using BayesTree. The SBART method is an extension of the BART algorithm, which involves a different specification of $w(\cdot)$. Public use code described in Linero and Yang (2018) is used.

\section{Appendix C.4: Choices of Basis Functions}

In Section 5, the $r$-dimensional real-valued vector $\mathbf{S}_{i j}$ is defined to be the Moran's I basis function (Hughes and Haran, 2013). The Moran's I basis functions (Griffith, 2000, $2002,2004)$ are motivated as a way to remove confounding between $\boldsymbol{\beta}$ and $\boldsymbol{\eta}$, and allow for dimension reduction. The basis functions are derived from the Moran's I operator used in spatial statistics (Moran, 1950). Specifically, basis functions are specified to be in the orthogonal column space associated with the hat matrix $\mathbf{X}\left(\mathbf{X}^{\prime} \mathbf{X}\right)^{-1} \mathbf{X}^{\prime}$, where the $N \times p$ matrix $\mathbf{X}=\left(\mathbf{x}_{i j}: i=1, \ldots, I, j=1,2,3\right)$. Define the Moran's I operator

$$
\mathbf{G}\left(\mathbf{X}, \mathbf{A}_{t}\right) \equiv\left(\mathbf{I}_{N}-\mathbf{X}\left(\mathbf{X}^{\prime} \mathbf{X}\right)^{-1} \mathbf{X}^{\prime}\right) \mathbf{W}\left(\mathbf{I}_{N}-\mathbf{X}\left(\mathbf{X}^{\prime} \mathbf{X}\right)^{-1} \mathbf{X}^{\prime}\right)
$$

where $\mathbf{W}$ is a generic real-valued $N \times N$ matrix, which is often specified to be an adjacency matrix that characterizes a network. The spectral representation $\mathbf{G}(\mathbf{X}, \mathbf{W})=$ $\boldsymbol{\Phi} \boldsymbol{\Lambda} \boldsymbol{\Phi}^{\prime}$, is computed using a $N \times N$ orthogonal matrix $\boldsymbol{\Phi}$ and a $N \times N$ diagonal matrix with positive elements $\Lambda$. Let the $N \times r$ real matrix consisting of the first $r$ columns of 


\section{Supplementary Materials: hierarchical generalized transformation model}

$\mathbf{\Phi}$ be denoted by $\mathbf{S}$. The row of $\mathbf{S}$ corresponding to the $(i, j)$-th data is set to equal to $\mathbf{S}_{i j}$. In Section 5, we set $r=500$.

In Section 6, the $r$-dimensional real-valued vector $\mathbf{S}_{i j}$ is defined to be thin-plate splines (Wahba, 1990). Specifically, let the $m$-th element of the 10-dimensional vector $\mathbf{S}_{i 1}^{(k)}$ be defined as,

$$
\left(t_{i} / 78-c_{m}\right)^{2} \log \left\{a b s\left(t_{i} / 78-c_{m}\right)\right\},
$$

where $c_{m}=\{0,0.11,0.22,0.33,0.44,0.56,0.67,0.78,0.89,1\}$ are 10 equally spaced values over $\left\{t_{1}, \ldots, t_{78}\right\}$. Then, let the $m$-th element of the 25 -dimensional vector $\mathbf{S}_{i j}^{*}$ be

$$
\left(t_{i} / 78-c_{m}^{*}\right)^{2} \log \left\{a b s\left(t_{i} / 78-c_{m}^{*}\right)\right\},
$$

where $\left\{c_{m}^{*}\right\}$ is a set of 25 equally spaced time-points between zero and one. Let the $\left|A_{k}\right| \times$ 10 matrix $\mathbf{S}_{1}^{(k)}=\left(\mathbf{S}_{i 1}^{(k)}: A_{i}=A_{k}\right)$ and the $I_{1} \times 2660$ matrix $\mathbf{S}_{1}=\operatorname{blkdiag}\left(\mathbf{S}_{1}^{(1)}, \ldots, \mathbf{S}_{1}^{(266)}\right)$, where blkdiag is the block-diagonal operator and $\left|A_{k}\right|$ is the number of observations recorded in region $A_{k}$ so that $I_{1}=\sum_{k}\left|A_{k}\right|$. Here, the $I_{1} \times 2660$ matrix $\mathbf{S}_{1}$ defines a set of basis matrices for each of the 266 regions in the study, and hence, we allow for different time series within each region. Note that some regions contain others (e.g., provinces are contained with countries). As such, shared time series within a country imply withincountry spatial dependence. Define the $I_{j} \times 25$ matrix $\mathbf{S}_{j}=\left(\mathbf{S}_{i j}^{*}: i=1, \ldots, I_{j}\right)$ for $j=2,3$, which defines basis matrices for each individual response-types. Then collect all individual-level basis matrices into the matrix $n \times 2710$ matrix $\mathbf{S}^{* *}=\operatorname{blkdiag}\left(\mathbf{S}_{1}, \mathbf{S}_{2}, \mathbf{S}_{3}\right)$. Let the $n \times 25$ matrix $\mathbf{S}^{*}=\left(\mathbf{S}_{i j}^{*}: i=1, \ldots, I_{j}, j=1,2,3\right)$, which represents the set of basis functions that are shared among all response-types. Finally, the $n \times 2735$ matrix $\mathbf{S}=\left(\mathbf{S}^{*}, \mathbf{S}^{* *}\right)$ represents the basis matrix used in our analysis, and the 2735 -dimensional $(i, j)$-th row is denoted with $\mathbf{S}_{i j}$.

\section{References}

Bradley, J. R., Holan, S. H., and Wikle, C. K. (2020+). "Bayesian Hierarchical Models with Conjugate Full-Conditional Distributions for Dependent Data from the Natural Exponential Family." Journal of the American Statistical Association. 4

Casella, G. and Berger, R. (2002). Statistical Inference. Pacific Grove, CA: Duxbury. 5

Chipman, H. and McCulloch, R. (2016). "BayesTree: Bayesian additive regression trees." $R$ package version 0.3-1.4. 7

Chipman, H. A., George, E. I., , and McCulloch, R. E. (2010). "BART: Bayesian additive regression trees." The Annals of Applied Statistics, 4(1): 266-298. 7

Cressie, N. and Wikle, C. K. (2011). Statistics for Spatio-Temporal Data. Hoboken, NJ: Wiley. 6

Diaconis, P. and Ylvisaker, D. (1979). "Conjugate priors for exponential families." The Annals of Statistics, 17: 269-281. 4, 5

Gelman, A., Carlin, J. B., Stern, H. S., Dunson, D. B., Vehtari, A., and Rubin, D. B. (2013). Bayesian Data Analysis, 3rd edn.. Boca Raton, FL: Chapman and Hall/CRC. 6 
Griffith, D. (2000). "A linear regression solution to the spatial autocorrelation problem." Journal of Geographical Systems, 2: 141-156. 7

- (2002). "A spatial filtering specification for the auto-Poisson model." Statistics and Probability Letters, 58: 245-251. 7

- (2004). "A spatial filtering specification for the auto-logistic model." Environment and Planning A, 36: 1791-1811. 7

Hughes, J. and Haran, M. (2013). "Dimension reduction and alleviation of confounding for spatial generalized linear mixed model." Journal of the Royal Statistical Society, Series B, 75: 139-159. 7

Lehmann, E. and Casella, G. (1998). Theory of Point Estimation. New York, NY: Springer, 2nd edition. 4

Linero, A. R. and Yang, Y. (2018). "Bayesian regression tree ensembles that adapt to smoothness and sparsity." Journal of the Royal Statistical Society: Series B (Statistical Methodology), 80(5): 1087-1110. 7

Moran, P. A. P. (1950). "Notes on Continuous Stochastic Phenomena." Biometrika, 37: $17-23.7$

Wahba, G. (1990). Spline Models for Observational Data. Philadelphia, PA: Society for Industrial and Applied Mathematics. 8

\section{Acknowledgments}

This research was partially supported by the U.S. National Science Foundation (NSF) under NSF grant SES-1853099. I also would like to thank Drs. Christopher Wikle and Scott Holan at the University of Missouri on their feedback on an earlier version of this article. 